\title{
Partial Anomalous Pulmonary Venous Return into the Inferior Vena Cava in a 28-Year-Old Female: A Variant of Scimitar Syndrome Amenable to Interventional Treatment
}

\section{Scimitar Syndrome Variant}

\author{
Jana-K. Dieks, MD*, Michael Steinmetz, MD, Thomas Paul, MD, Heike E. Schneider, MD \\ Department of Pediatric Cardiology and Intensive Care Medicine, University Hospital, Georg-August-University Göttingen, Germany
}

\begin{abstract}
Scimitar syndrome is a rare congenital heart defect associated with right-sided partial anomalous pulmonary venous return (PAPVR) into the inferior vena cava (IVC). We describe the case of a 28-year-old female diagnosed with PAPVR with a typical curvilinear pattern of the right lower pulmonary vein-the so-called "scimitar sign" - on chest x-ray. Anatomical abnormalities on magnetic resonance imaging included a lower right pulmonary vein draining into the IVC and an additional vein connecting all right-sided pulmonary veins toward the left atrium. Because her anatomy was suitable, the patient underwent cardiac catheterization with occlusion of the anomalous right lower pulmonary vein with an Amplatzer Vascular Plug with excellent postinterventional results.

Copyright @ 2016 Science International Corp.
\end{abstract}

\section{Key Words}

PAPVR • Amplatzer vascular plug • Catheter intervention - Congenital heart disease

\section{Introduction}

The term "scimitar syndrome" was first employed by Neill et al. in 1960 to describe a rare type of partial anomalous pulmonary venous return (PAPVR) in

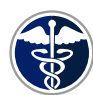

Fax +1 2037853346

E-Mail: jshd@scienceinternational.org

http://structuralheartdisease.org/ (c) 2016 Journal of Structural Heart Disease Published by Science International Corp. ISSN 2326-4004

Accessible online at:

http://structuralheartdisease.org/ combination with a hypoplastic right lung that receives its blood supply from systemic arteries [1]. Associated features include dextroposition of the heart, bronchopulmonary sequestration, and various extracardiac and additional cardiac anomalies. The classic radiographic finding is the "scimitar sign" on chest $x$-ray resulting from the curved anomalous right pulmonary vein draining into the inferior vena cava (IVC). Two forms of scimitar syndrome can be differentiated. In the infantile variant, patients usually present with tachypnea, heart failure, and pulmonary hypertension, and significant morbidity and mortality $[2,3]$. In the childhood/adult form, patients are less severely affected and may be asymptomatic until a diagnosis is established. The adult variant of scimitar syndrome is commonly associated with right lung hypoplasia, abnormalities of vascular supply, dextrocardia, and abnormalities of bronchial segmentation [4]. Treatment generally implies a surgical approach, and procedural details vary depending on individual anatomic and pathologic features, as well the surgeon's preference. Procedures frequently include intra-atrial baffle repair and reimplantation of the scimitar vein into the left atrium and, if necessary, simultaneous ligation of collateral arteries supplying the right lung, right lower lobe

* Corresponding Author:

Jana-K. Dieks, MD

University Hospital, Georg-August University Göttingen

Department of Pediatric Cardiology and Intensive Care Medicine

Robert-Koch Str. 40, 37075 Göttingen, Germany

Tel.: +49 5513966203 ; Fax: +49 55139 22561; E-Mail: jana.dieks@med.uni-goettingen.de 
resection, or even right pneumonectomy [5]. Successful attempts of interventional therapy of scimitar syndrome include transcatheter embolization of anomalous systemic arterial supply with interventional atrial septal defect closure [6] and rerouting of anomalous venous drainage to the left atrium [7].

\section{Case Report}

We present a 28-year-old female who was diagnosed with right-sided PAPVR as a variant of scimitar syndrome. The patient had been a preterm infant born at 26 weeks of gestation. She developed bronchopulmonary dysplasia and suffered from recurrent respiratory tract infections in infancy and early childhood. Her psychomotor and physical development were unremarkable. At the age of 28 years, the patient presented to our unit with exertional dyspnea. On chest $x$-ray, a tubular structure in the right cardiophrenic angle compatible with the typical radiologic sign of scimitar syndrome was evident (Figure 1). Subsequent MRI revealed anomalous return of the right lower pulmonary vein into the IVC. The scimitar vein was slightly stenotic proximal

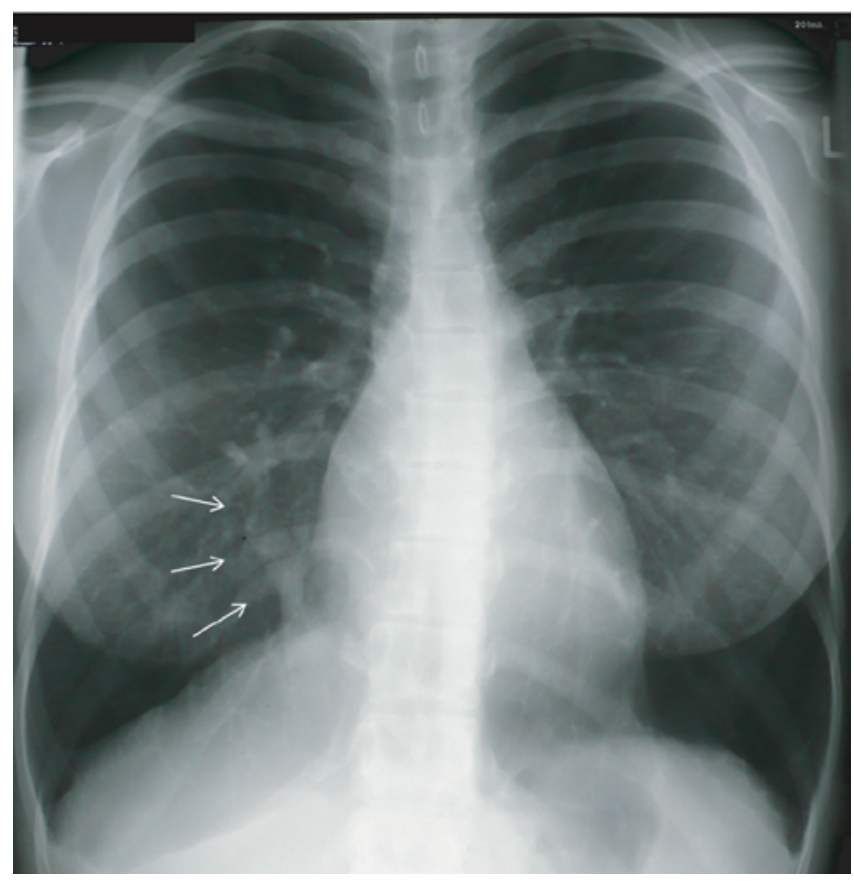

Figure 1. Chest x-ray with a classic "scimitar sign" resulting from a right pulmonary vein coursing to the right cardiophrenic angle (arrows). to its drainage into the dilated IVC. Furthermore, all right-sided pulmonary veins were connected via the scimitar vein and drained not only into the IVC but also into the left atrium, thus representing a rare variant of scimitar syndrome. The right pulmonary veins had a more tortuous appearance than usual, but no discrete stenosis was noted (Figure 2). The right ventricle was enlarged due to volume overload, and systolic function was within normal limits. The pulmonary to systemic flow (Qp:Qs) ratio, as assessed by MRI, was 1.4:1.

Cardiac catheterization was performed with the intention of interventional closure of the aberrant pulmonary vein. Levophase after right pulmonary angiography confirmed dual connection of all right pulmonary veins via the scimitar vein to the IVC, as well as to the left atrium (Figure 3A). The left-to-right shunt ratio (according to Fick) was calculated to be 1.6:1. The patient's right ventricular systolic pressure

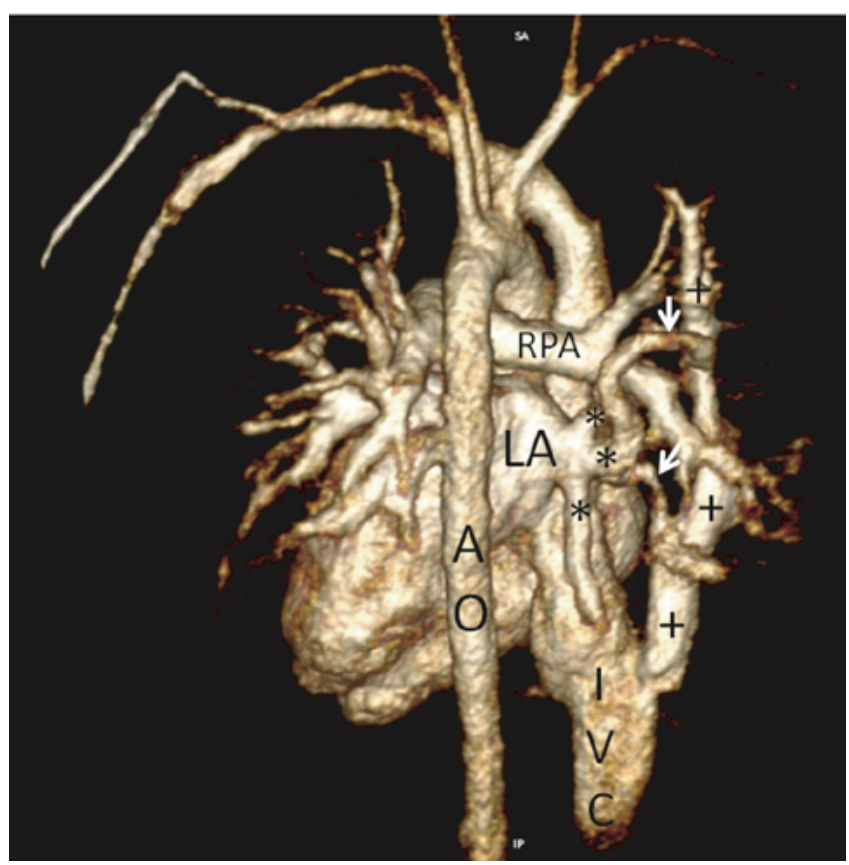

Figure 2. Three-dimensional reconstruction of cardiac MRI (posterior-anterior view). The anomalous drainage of the lower pulmonary right vein into the IVC with slight stenosis of the vessel at the diaphragm is evident. In addition, all three right pulmonary veins are connected to the left atrium. $\mathrm{AO}=$ descending aorta; $I V C=$ inferior vena cava; $L A=$ left atrium; RPA = right pulmonary artery; ${ }^{*}$ pulmonary veins; + scimitar vein; arrows, additional vein connecting the lower and middle right pulmonary vein. 

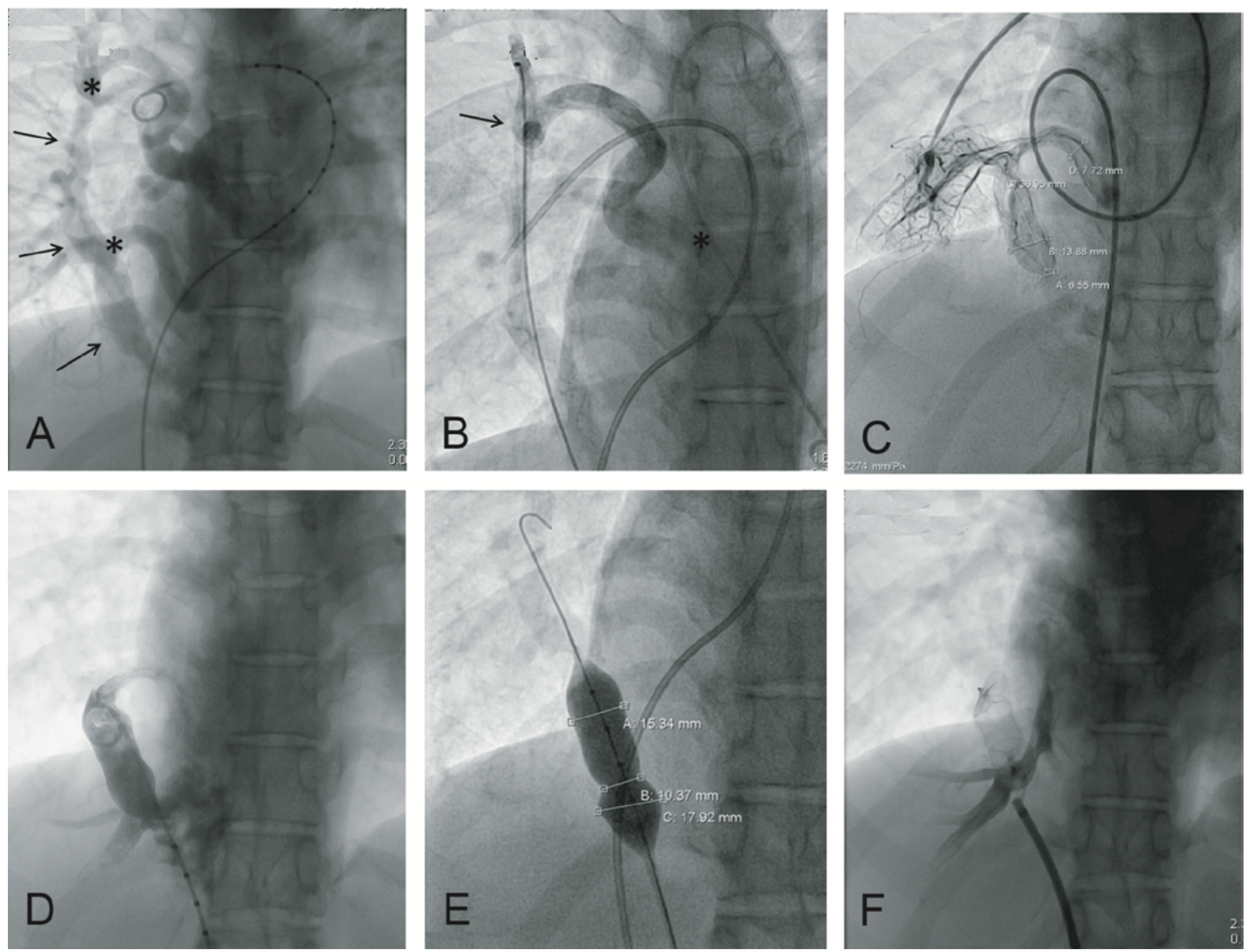

Figure 3. Cardiac catheterization (anterior-posterior views). Panel A. Levophase after right pulmonary angiography demonstrates typical curvilinear pattern (arrows), the so-called "scimitar sign." Drainage of the upper and middle right pulmonary veins into this descending vein (asterisks) connected to the inferior vena cava (IVC) is evident. Panel B. A guide catheter was directed from the IVC into the scimitar vein; hand injection of contrast demonstrates free drainage of the right upper pulmonary vein into this descending vein (arrow) and the left atrium (asterisk). Panel C. Diameter of the connecting vein is $13.9 \mathrm{~mm}$, stenosis before drainage to the IVC $6.6 \mathrm{~mm}$. Panel D. The lower part and stenosis of the anomalous vein are documented by direct angiography. Panel E. Test balloon occlusion of the lower connecting vein. Panel F. After implantation of an Amplatzer Vascular Plug II into the lower part of the scimitar vein, hand injection through the delivery sheath demonstrates no obstruction of the hepatic venous flow into the IVC.

was slightly elevated at $34 \mathrm{mmHg}$, and the mean pulmonary arterial pressure was $26 \mathrm{mmHg}$ with an indexed pulmonary vascular resistance of $3.0 \mathrm{WE} \times \mathrm{m}^{2}$ and a pulmonary to systemic vascular resistance (Rp:Rs) ratio of 0.19 . No pulmonary vasodilator studies were performed in the cardiac catheterization lab. The right pulmonary veins were tortuous without any obvious obstruction (Figure 3B). Angiography of the descending aorta did not reveal evidence of aortopulmonary collateral arteries. Balloon wedge angiography into the right lower pulmonary artery delineated the lower part of the scimitar vein in detail, demonstrating moderate stenosis just below the diaphragm (Figure 3C, D). Balloon occlusion of the vessel did not result in elevation of pulmonary right venous pressure or left ventricular end-diastolic 
pressure, indicating that interventional occlusion was feasible. During balloon occlusion, the diameter of the connecting vein was $15.3 \mathrm{~mm}$, with a stenotic segment of $10.4 \times 12.7 \mathrm{~mm}$ (Figure 3E). Subsequently, a 20-mm Amplatzer Vascular Plug II (AVP II) was chosen to occlude this portion of the scimitar vein. The stenosis was used as the anchor for the distal waist of the device. After release from the delivery system, there was no residual shunt through the device after $10 \mathrm{~min}$. Absence of obstruction of hepatic venous flow into the IVC and of the right lower pulmonary vein could be documented (Figure 3F). Repeat hemodynamic evaluation did not reveal any difference of pulmonary wedge pressure to the end-diastolic left ventricular pressure. However, the mean right pulmonary artery pressure remained elevated at $26 \mathrm{mmHg}$.

Echocardiography postimplantation showed no residual flow along the vessel occluder into the IVC. Before hospital discharge, the patient was started on a vitamin $\mathrm{K}$ antagonist for 6 months. No oral pulmonary vasodilator therapy was initiated after the intervention. At the 6-month follow-up, the patient was well, and the device was in an unchanged position without residual flow as assessed by echocardiography. Repeat cardiac catheterization 19 months after the intervention revealed improved hemodynamics with a slightly increased mean pulmonary artery pressure at $18 \mathrm{mmHg}$, underscoring the need for anomalous vessel closure.

\section{Discussion}

Unless patients diagnosed with scimitar syndrome are symptomatic or have a significant left-to-right shunt leading to pulmonary hypertension and right ventricular failure, clinical surveillance has been recommended, because surgery may be challenging and associated with significant complications and morbidity [8-10]. In the present case, moderate leftto-right-shunting and mild pulmonary hypertension required treatment. There was partial anomalous pulmonary venous drainage of the right lung into the IVC without further abnormalities of the right lung, including blood supply. The patient's individual anatomy with double drainage of the right lung allowed successful interventional occlusion of the inferior portion of the scimitar vein without open surgery.

Unfortunately, very few patients diagnosed with classical scimitar syndrome fulfill anatomic requirements for catheter-based rerouting of the anomalously draining vein. This case illustrates that interventional therapy should be considered and may be successful in selected patients with unusual anatomical variants.

\section{Conclusion}

Particular attention to anatomic variants of scimitar syndrome may identify novel interventional treatment options.

\section{Conflict of Interest}

The authors have no conflict of interest relevant to this publication.

\section{Comment on this Article or Ask a Question}

\section{References}

1. Neill CA, Ferencz C, Sabiston DC, Sheldon H. The familial occurrence of hypoplastic right lung with systemic arterial supply and venous drainage "scimitar syndrome." Bull Johns Hopkins Hosp. 1960;107:1-21. PMID: 14426379

2. Gao YA, Burrows PE, Benson LN, Rabinovitch $M$, Freedom RM. Scimitar syndrome in infancy. J Am Coll Cardiol. 1993;22:873882. DOI: 10.1016/0735-1097(93)90206-G

3. Huddleston CB, Exil V, Canter CE, Mendeloff EN. Scimitar syndrome presenting in infancy. Ann Thorac Surg. 1999;67:154159; discussion 160. DOI: 10.1016/S0003-
4975(98)01227-2

4. Dupuis $C$, Charaf LA, Brevière GM, Abou $P$, Rémy-Jardin M, Helmius G. The "adult" form of the scimitar syndrome. Am J Cardiol. 1992;70:502-507. DOI: 10.1016/00029149(92)91198-D

5. Vida VL, Padalino MA, Boccuzzo G, Tarja E, Berggren $H$, Carrel $T$, et al. Scimitar syndrome: a European Congenital Heart Surgeons Association (ECHSA) multicentric study. Circulation. 2010;122:11591166. DOI: 10.1161/CIRCULATIONAHA.109.926204
6. Noble S, Miró J, Ibrahim R. Transcatheter embolization of anomalous systemic arterial supply with Amplatzer Vascular Plugs II in Scimitar syndrome. Catheter Cardiovasc Interv. 2012;80:477-481. DOI: 10.1002/ccd.23329

7. Singh $H$, Luthra $M$, Bharadwaj $P$, Kumar $R$. Interventional rerouting of scimitar vein to left atrium using an Amplatzer vascular plug. Congenit Heart Dis. 2007;2:265-269. DOI: 10.1111/j.1747-0803.2007.00113.x

8. Honey M. Anomalous pulmonary venous drainage of right lung to inferior vena cava 
("scimitar syndrome"): clinical spectrum in older patients and role of surgery. Q J Med. 1977;46:463-483. PMID: 339257

9. Najm HK, Williams WG, Coles JG, Rebeyka IM, Freedom RM. Scimitar syndrome: twenty years' experience and results of repair. J Thorac Cardiovasc Surg. 1996;112:11611168; discussion 1168-1169. DOI: $10.1016 /$ S0022-5223(96)70129-0
10. Schramel FM, Westermann CJ, Knaepen PJ, van den Bosch JM. The scimitar syndrome: clinical spectrum and surgical treatment. Eur Respir J. 1995;8:196-201. DOI: 10.1183/09031936.95.08020196
Cite this article as: Dieks J-K, Steinmetz M, Paul T, Schneider HE. Partial Anomalous Pulmonary Venous Return Into the Inferior Vena Cava in a 28-Year-Old Female: A variant of Scimitar Syndrome Amenable to Interventional Treatment. Structural Heart Disease. 2016;2(5):208-212. DOI: http://dx.doi.org/10.12945/j.jshd.2016. 013.15 